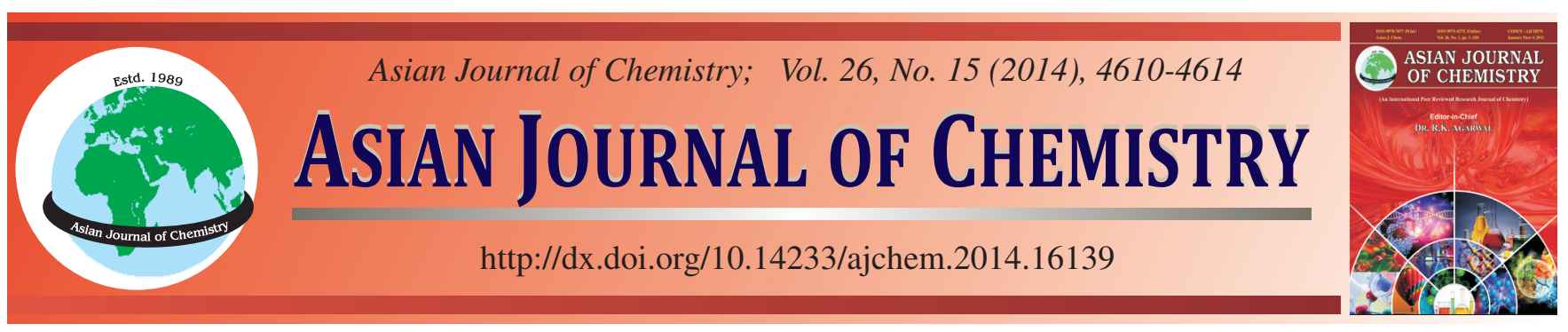

\title{
Studies on Purification Process of Total Saponins in Radix Astragali with Resin and Structural Identification of Compounds
}

\author{
Shan-Bin Guo, XiaO-Ming Du and Ling-Yan JiAn ${ }^{*}$
}

Department of Pharmacy, Shengjing Hospital of China Medical University, Shenyang, P.R. China

*Corresponding author: Tel: +86 24 9661571101; E-mail: jianlingyan126@126.com

\begin{abstract}
This study reports the process conditions for purification and enrichment of total saponins in Radix Astragali with macroporous adsorption resin. Reflux method was used to obtain Radix Astragali extract; spectrophotometry was used to determine the content of total saponins in Radix Astragali and to study the selection of macroporous adsorption resin types as well as purification conditions. D101 resin had a relatively strong static adsorption-desorption capacity. The enrichment and purification effects of total saponins in Radix Astragali were optimal when the concentration of total saponins of Radix Astragali was selected as $40 \mathrm{mg} / \mathrm{mL}$, sample adsorption rate was selected as 3 $\mathrm{BV} / \mathrm{h}$, adsorption column with a diameter height ratio of 1:10 was used and $80 \%$ ethanol was used as the eluent; $\mathrm{pH}$ value of drug solution had no effect on the results. Under the experimental conditions, D101 resin is suitable for the extraction, isolation and purification of total saponins in Radix Astragali.
\end{abstract}

Keywords: D101 resin, Total saponins in Radix Astragali, Astragaloside.

\section{INTRODUCTION}

Radix Astragali is the root of Astragalus membranaceus (Fisch.) Bge. var. mongholicus (Bge.) Hsiao or Astragalus membranaceus (Fisch.) of family Leguminosae ${ }^{1}$. Radix Astragali contains a variety of substances such as glycosides, polysaccharides, flavonoids, amino acids, trace elements, etc. ${ }^{2-4}$, which has antitumor, immunomodulatory, antiviral, antiaging, antioxidant, antiradiation, antistress and other pharmacological effects $^{5,6}$. Macroporous adsorption resin is a high polymer adsorbent with a macroporous structure which does not contain exchange groups, it itself has adsorption capacity due to van der Waals force or hydrogen bonding interactions, it also has screening capacity due to its mesh structure and high specific surface area.

In recent years, macroporous adsorption resin has been used increasingly in the studies on extraction and purification of traditional Chinese medicines ${ }^{7,8}$. This study investigates the effects of macroporous adsorption resins with different polarities on adsorption performance of total saponins in Radix Astragali and explores the effects of drug solution, adsorbent and adsorption column on the adsorption-desorption effect of total saponins in Radix Astragali, meanwhile, the enriched and purified compounds of Radix Astragali total saponins are isolated and identified.

EXPERIMENTAL

Model 722 spectrophotometer (Shandong High-Density Analytical Instrument Factory), model HH420-3C water bath (Shanghai Bilon Instrument Co., Ltd.); model AB204-B electronic analytical balance (Mettler, Switzerland), all the reagents used were of analytical grade. Radix Astragali was purchased from Dongbei Pharmacy, which was identified as Astragalus membranaceus (Fisch.) Bge. var. mongholicus (Bge.) Hsiao.

Determination of total saponins in Radix Astragali: $2.5 \mathrm{mg}$ of Astragaloside reference substance which was dried to constant weight was precisely weighed, added with methanol to make the volume $10 \mathrm{~mL}$, then $0,0.2,0.4,0.6,0.8,1.0$ and $1.2 \mathrm{~mL}$ of the solution were precisely drawn, respectively and evaporated to dryness in water bath, added with $5 \%$ vanillin glacial acetic acid solution $(0.2 \mathrm{~mL})$ and perchloric acid $(0.8 \mathrm{~mL})$ and heated in a $65^{\circ} \mathrm{C}$ water bath for $20 \mathrm{~min}$, removed, cooled, added with $10 \mathrm{~mL}$ of glacial acetic acid and shaken uniformly, followed by measurement of absorbance at 560 $\mathrm{nm}$. Standard curves were prepared and regression equation was obtained as $\mathrm{Y}=0.034 \times-0.0312, \mathrm{R}=0.9996$, the linear relationship was good.

Selection of extraction method: $30 \mathrm{~g}$ of crude Radix Astragali powder was weighed in triplicate and extracted with various solvents (water, $50 \%$ ethanol and $80 \%$ ethanol) as 
follows: crude Radix Astragali powders were soaked in 8-fold amount of three solvents for $0.5 \mathrm{~h}$ and reflux extracted three times, each time lasted $2 \mathrm{~h}$. The extracts were then combined, concentrated to $20 \mathrm{~mL}$, respectively and the content of Radix Astragali total saponins was measured. Results showed that the extraction efficiencies of three solvents were almost equivalent. The content of Radix Astragali total saponins was slightly lower for water extraction than for ethanol extraction. Considering factors such as actual mass production, energy conservation and environmental protection, water extraction was chosen.

Preparation of sample solution: $5 \mathrm{~kg}$ of Radix Astragali was taken, ground into coarse powder, added with an 8-fold amount of water and soaked for $0.5 \mathrm{~h}$, then reflux extracted three times, each time lasted $2 \mathrm{~h}$, the extracts were then combined and concentrated to give Radix Astragali extract. Radix Astragali extract (containing 58.98\% Radix Astragali total saponins) was weighed, added with water and ultrasonically dissolved for $15 \mathrm{~min}$, then filtered, the filtrate was taken and set aside for later use.

\section{Selection of resin type}

Pretreatment of resins: Resins were soaked in $95 \%$ ethanol for $24 \mathrm{~h}$, after fully swollen, the resins were washed with distilled water till no ethanol was left. The resins were then soaked in 3 column volumes (BV) of $5 \%$ hydrochloric acid for $12 \mathrm{~h}$, washed with water to nearly neutral and then soaked in $5 \%$ sodium hydroxide solution for $12 \mathrm{~h}$, washed with water to nearly neutral and filtered and set aside for later use.

Static adsorption-desorption test: About $10 \mathrm{~g}$ of pretreated resins were weighed, placed in $100 \mathrm{~mL}$ beaker, added with $25 \mathrm{~mL}$ of sample solution and set aside for $24 \mathrm{~h}$ so that the resin reached saturated adsorption, then filtered, the amount of filtrate was precisely weighed and the concentration of Radix Astragali total saponins was determined. The saturated adsorption capacity of resin was calculated, saturated adsorption capacity $=[$ (initial concentration-concentration after adsorption) $\times$ adsorption volume]/resin mass; the concentration of desorption solution was determined and the desorption rate was calculated. Desorption rate $=($ concentration of desorption solution $\times$ volume of desorption solution $\div$ resin mass $)$ / saturated adsorption capacity $\times 100 \%$, the results are shown in Table-1 (Fig. 1).

The experimental results showed that the static adsorptiondesorption capacity on Radix Astragali total saponins varied for macroporous adsorption resins with different polarities,

\begin{tabular}{cccc}
\multicolumn{4}{c}{ TABLE-1 } \\
& $\begin{array}{c}\text { STATIC SATURATED ADSORPTION } \\
\text { CAPACITY OF DIFFERENT RESINS }(\mathrm{N}=4)\end{array}$ \\
\hline $\begin{array}{c}\text { Resin } \\
\text { type }\end{array}$ & $\begin{array}{c}\text { Drug } \\
\text { oncentration } \\
(\mathrm{mg} / \mathrm{mL})\end{array}$ & $\begin{array}{c}\text { Concentration } \\
\text { after adsorption } \\
(\mathrm{mg} / \mathrm{mL})\end{array}$ & $\begin{array}{c}\text { Saturated dsorption } \\
\text { capacity } \\
(\mathrm{mg} / \mathrm{mL})\end{array}$ \\
\hline D-101 & 7.24 & 1.89 & 13.68 \\
HP-20 & 7.24 & 1.45 & 10.49 \\
XDA-5 & 7.24 & 1.67 & 12.09 \\
AB-8 & 7.24 & 1.85 & 11.44 \\
ASD-7 & 7.24 & 1.49 & 10.79 \\
S-8 & 7.24 & 1.64 & 11.87
\end{tabular}
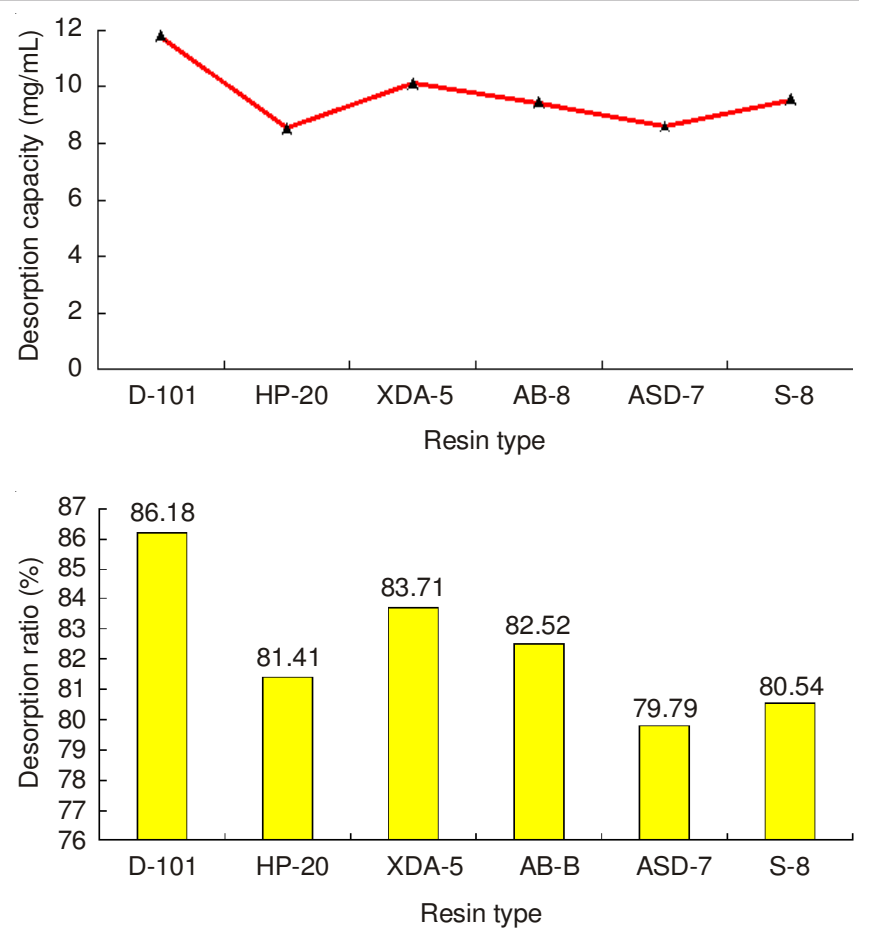

Fig. 1. Static adsorption-desorption rate of different resins $(n=4)$

yet the results were not significant. By comprehensive comparison, D101 resin had a relatively strong static adsorptiondesorption capacity, so D101 resin was used in the test.

\section{Experiment on purification conditions with adsorption resin}

Investigation on concentration of column-loading drug solution: Radix Astragali total saponins solutions (concentrations of 10, 20, 40, 80 and $160 \mathrm{mg} / \mathrm{mL}$ ) were passed through the pretreated D101 resin, respectively, for dynamic adsorption and the concentration of Radix Astragali total saponins was determined.

Fig. 2 showed that at the same rate, the same pore size and the same drug-resin mass ratio, low concentrations of Radix Astragali total saponins (10, 20 and $40 \mathrm{mg} / \mathrm{mL}$ ) had relatively good absorption effect, compared with low concentrations, more Radix Astragali total saponins were eluted for the high concentrations of Radix Astragali total saponins under the same conditions due to higher concentrations and absorbance values increased significantly. Combined with practice and taking full account of large-scale production, the concentration of Radix Astragali total saponins was selected as 40 $\mathrm{mg} / \mathrm{mL}$.

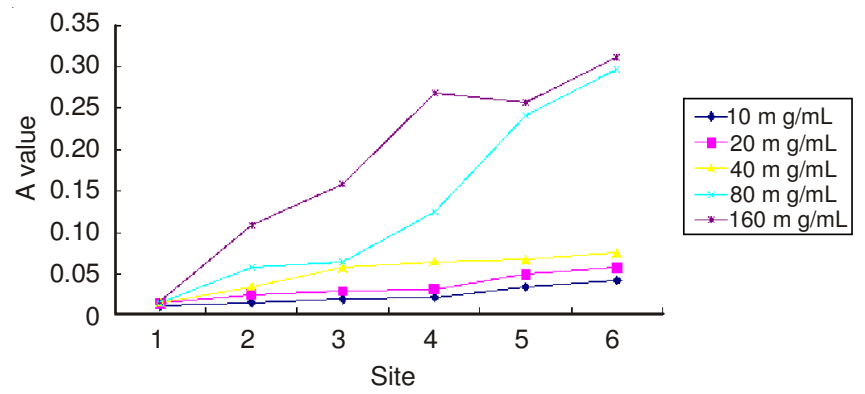

Fig. 2. Effects of different concentrations of Radix Astragali total saponins on adsorption capacity of D101 resin 
Investigation on $\mathrm{pH}$ value of column-loading drug solution: $10 \mathrm{~mL}$ of Radix Astragali total saponins with a concentration of $40 \mathrm{mg} / \mathrm{mL}$ were taken in quadruplicate, of which three portions were $\mathrm{pH}$ adjusted to 6,9 and 12, respectively and dynamically adsorbed at the same rate with the same mass of D101 resin, then eluted with $95 \%$ ethanol, followed by the determination of concentration of Radix Astragali total saponins. The results showed that compared with the original drug solution, the content of Radix Astragali total saponins decreased slightly for the $\mathrm{pH}$ adjusted Radix Astragali total saponins solutions, yet the differences were not statistically significant, therefore, in practical operation, adjustment of the $\mathrm{pH}$ value of the drug solution was not needed.

Effect of sample adsorption rate on adsorption capacity of resin: $40 \mathrm{mg} / \mathrm{mL}$ sample solution was taken for performing resin adsorption experiment at the flow rates of 1 , 2, 3, 4 and $5 \mathrm{BV} / \mathrm{h}$. The mass concentration of sample solution after adsorption was measured, the adsorption amount of Radix Astragali total saponins by D101 resin under different rates was calculated and the adsorption curves were prepared (Fig. 3).

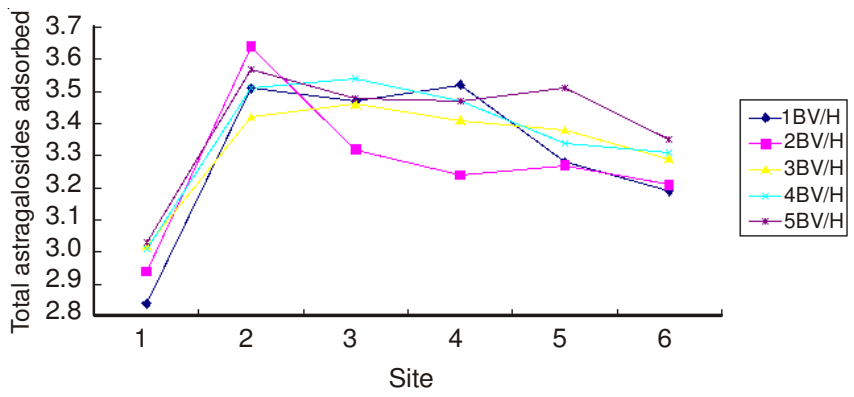

Fig. 3. Effect of different rates on content of Radix Astragali total saponins

As can be seen from the adsorption curves, the slower the rate, the better the adsorption effect, yet the longer the time expanded and the lower the efficiency. The differences in the content of Radix Astragali total saponins under various efficiency conditions were not statistically significant, comprehensively considering various factors, $3 \mathrm{BV} / \mathrm{h}$ was selected as the sample adsorption rate.

Investigation on eluent concentration: $10 \mathrm{~g}$ of pretreated D101 resin was taken, $20 \mathrm{~mL}$ of Radix Astragali total saponins (concentration of $40 \mathrm{mg} / \mathrm{mL}$ ) was loaded on the column and eluted with 10, 20, 40, 80 and $95 \%$ ethanol and anhydrous ethanol at a flow rate of $3 \mathrm{BV} / \mathrm{h}$, respectively, the concentration of Radix Astragali total saponins was measured and elution rate was calculated.

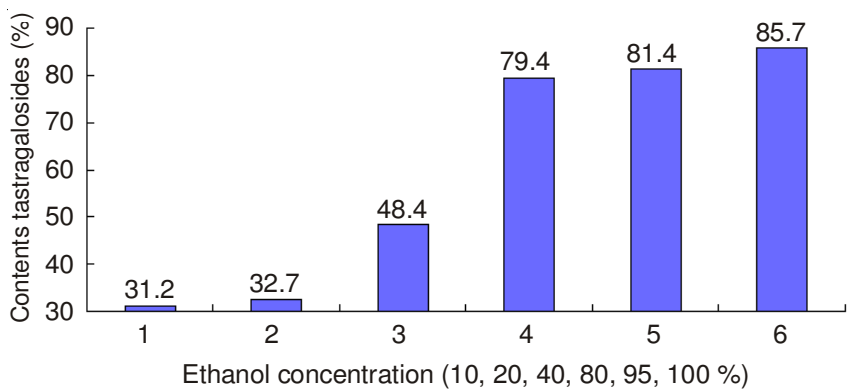

Fig. 4. Results for elution of Radix Astragali total saponins using different concentrations of ethanol
As can be seen from Fig. 4, the elution efficiency of high concentrations of ethanol (80, 95 and $100 \%)$ was significantly higher than low concentrations of ethanol, taking into account the mass production safety, cost saving and other factors, $80 \%$ ethanol was used as the eluent.

Investigation on diameter height ratio of resin: $50 \mathrm{~mL}$ of Radix Astragali total saponins $(40 \mathrm{mg} / \mathrm{mL})$ was taken and passed through D101 resin columns with different pore sizes at a flow rate of $3 \mathrm{BV} / \mathrm{h}$, then eluted with $300 \mathrm{~mL}$ of $80 \%$ ethanol, the ethanol eluents was collected and the content of Radix Astragali total saponins was determined, the results are shown in Fig. 5.

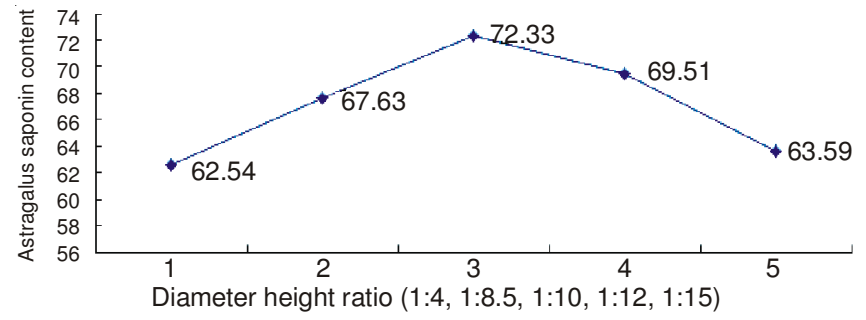

Fig. 5. Effect of diameter height ratio of adsorption column on the content of Radix Astragali total saponins

As can be seen from Fig. 5, the diameter height ratio has a relatively obvious effect on the experimental results. After the same volume of macroporous adsorption resins were added to adsorption columns with different diameter height ratios, adsorption columns with too large diameter height ratios had relatively short paths and relatively small adsorbed drug solutions; adsorption columns with too small diameter height ratios were relatively thin and had longer paths and poor desorption effects by the desorption solution. The experimental results showed that the adsorption column with a diameter height ratio of 1:10 had the best adsorption performance, which reached $72.33 \%$.

Extraction, purification and isolation of Radix Astragali total saponins and compound identification

Enrichment and purification of total saponins in Radix Astragali with macroporous adsorption resin: The conditions for enrichment and purification of total saponins in Radix Astragali were in accordance with the results of this experiment: $50 \mathrm{~g}$ of Radix Astragali total saponins was taken, prepared into a $40 \mathrm{mg} / \mathrm{mL}$ solution, passed through the pretreated D-101 macroporous adsorption resin column and eluted with $80 \%$ ethanol at a flow rate of $3 \mathrm{BV} / \mathrm{h}$, according to the actual experiment, the diameter height ratio of adsorption column was controlled at 1:10 as far as possible. Desorption solution was removed and the same fractions were combined. Three compounds were isolated therefrom by column chromatography, preparative TLC and preparative liquid chromatography.

Compound 1: White powders, the above data was basically consistent with the literature ${ }^{3}$. The structure of compound 1 was Astragaloside.

Compound 2: White needle crystals, easily soluble in methanol, m.p. $206-208^{\circ} \mathrm{C}$ (decomposition). The above spectral data were all consistent with the (6aR,11aR)-9,10-dimethoxypterocarpan-3-O- $\beta-\mathrm{D}$ glucoside reported in the literature ${ }^{9}$. 

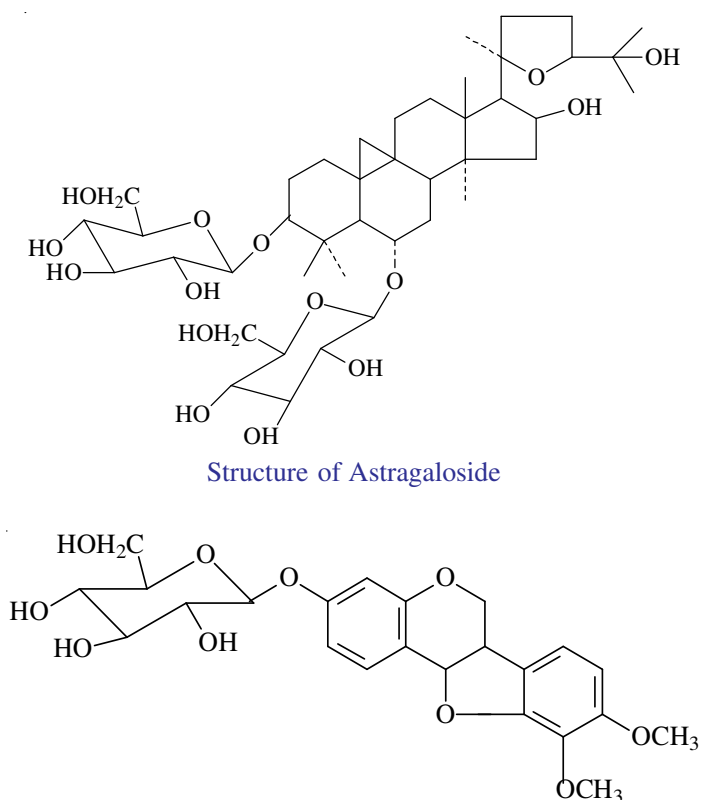

Structure of (6aR,11aR)-9,10-dimethoxypterocarpan-3-O- $\beta$-D glucoside

Compound 4: colourless needle crystals (methanol), m.p. $151{ }^{\circ} \mathrm{C}$, easily soluble in methanol. Comparing the above data with the literature ${ }^{10}$, compound 4 was identified as (3R)-8,2'dihydroxy-7,4'-dimethoxyisoflavan.<smiles>COc1ccc(C2COc3c(ccc(OC)c3O)C2)c(O)c1</smiles>

Structure of (3R)-8,2'-dihydroxy-7,4'-dimethoxyisoflavan

\section{RESULTS AND DISCUSSION}

Macroporous adsorption resin is a new class of high polymers, which has three-dimensional pore structure, relatively large pore size and specific surface area and a screening capacity $^{11}$. Macroporous adsorption resin has the advantages of stable physical and chemical properties, unique adsorption selectivity, mild desorption conditions, simple regeneration and long life cycle ${ }^{12-14}$ and has been widely used in the extraction, isolation, purification and enrichment of effective parts and monomer chemical components in single and compound traditional Chinese medicines ${ }^{15,16}$, providing a new way for the research and development of Chinese medicines and their compound preparations.

There are many factors influencing the adsorption and isolation effects of macroporous adsorption resins, which mainly include the structure of resin, structure of isolated compound, concentration and $\mathrm{pH}$ value of column-loading solution, ambient temperature, etc. Generally speaking, the polarity and spatial structure (mainly pore size, specific surface area, pore volume, etc.) of the resin are important influential factors of the adsorption performance. Non-polar compounds can be adsorbed in water by non-polar resins, while the polar resins can easily adsorb polar substances in water. Under a certain conditions, the greater the volume of the compound, the stronger the adsorption force.

Macroporous adsorption resins can be divided into polar, neutral and non-polar these three categories based on different skeleton materials ${ }^{11}$. Macroporous adsorption resin can, by adsorption and screening properties, isolate organic compounds by eluting them through resins using certain solvents according to the differences in adsorption force and molecular weights. In order to fully consider the differences in Radix Astragali total saponins adsorption performance among various types of resins, this experiment used six types of resins, namely D-101, HP-20, XDA-5, AB-8, ASD-7 and S-8, which basically covered all resin types. The experimental results showed that the non-polar D101 resin had a relatively strong static adsorption-desorption capacity.

There are a variety of factors influencing the adsorption performance of macroporous adsorption resin, this paper firstly examined the influences of concentration and $\mathrm{pH}$ value of column-loading drug solution (Radix Astragali total saponins). The concentration of column-loading drug solution influenced the adsorption amount of effective constituents by macroporous adsorption resin. Too high concentration of loading solution will lead to premature leakage of the effective constituents, which is a waste of medicinal materials; if the concentration of loading solution is too low, the purification time will increase, reducing the efficiency of the experiment. Under the experimental conditions, the adsorption performance of 10, 20, 40, 80 and $160 \mathrm{mg} / \mathrm{mL}$ concentrations of Radix Astragali total saponins varied slightly after loading. At the same rate, the same pore size and the same drug-resin mass ratio, low concentrations of Radix Astragali total saponins (10, 20 and 40 mg/ $\mathrm{mL}$ ) had relatively good adsorption effects, compared with low concentrations, more Radix Astragali total saponins were eluted and lost for the high concentrations of Radix Astragali total saponins under the same conditions due to higher concentrations and the adsorption effects were poor. The experiment on the investigation of $\mathrm{pH}$ value of column-loading drug solution found that, within the range of $\mathrm{pH}$ where the nature of drug solution does not change, the adsorption performance of resin was affected little under acidic or alkaline conditions.

Selection of eluent should be determined based on the resin's adsorption capacity on index components, in general, for the non-polar and weakly polar macroporous resins, the smaller the polarity of the eluent, the stronger the elution capacity; for medium polar macroporous resins and highly polar compounds, highly polar solvent is more appropriate. Common types of eluents include methanol, ethanol, acetone and ethyl acetate. Taking into account the feasibility in the production, this study explored the elution effect of different concentrations of ethanol on Radix Astragali total saponins. It was found during the elution with 10, 20, 40, 80 and $95 \%$ ethanol and anhydrous ethanol that, elution efficiency of high concentrations of ethanol (80, 95 and $100 \%)$ was significantly higher than low concentrations of ethanol, considering the mass production safety and cost savings and other factors, $80 \%$ ethanol was used as the eluent.

Under normal conditions, the faster the adsorption velocity, the smaller the amount of drug adsorption by resin; if the 
elution flow rate is fast, the eluting solvent will flow out from the column before it can exchange and dissolve the effective constituents adsorbed on the resin, thus unable to achieve the isolation and purification purposes. If the flow rate is too slow, too much time will be consumed on the experiment. Therefore, appropriate adsorption and elution flow rates should be chosen. The results of this experiment showed that the slower the flow rate, the better the absorption effect, yet the longer the time exptended and the lower the efficiency. Under each efficiency conditions, the change in the content of Radix Astragali total saponins was not statistically different, comprehensively considering various factors, $3 \mathrm{BV} / \mathrm{h}$ was selected as the sample adsorption rate.

After careful survey on the effects of various factors on purification and enrichment of Radix Astragali total saponins, this paper isolated and purified total saponins in Radix Astragali based on the optimal conditions investigated and performed structural identification on the obtained compounds, three compounds were isolated, which were astragaloside, (6aR,11aR)9,10-dimethoxypterocarpan-3-O- $\beta$-D glucoside and (3R)-8,2'dihydroxy-7,4'-dimethoxyisoflavan, respectively.

\section{REFERENCES}

1. Jiangsu New Medical College, Dictionary of Chinese Medicine, pp. 2036-2040 (1997).

2. Z.Z. Cao, J.H. Yu, L.X. Gan and Y.Q. Chen, Acta Chim. Sinica, 43, 581 (1985).

3. M. Hirotani, Y. Zhou, H. Lui and T. Furuya, Phytochemistry, 36, 665 (1994).

4. M. Hirotani, Y. Zhou, H. Rut and T. Furuya, Phytochemistry, 37, 1403 (1994).

5. Hand book of Flora Medicine Effective Constituents, p. 586 (1986).

6. W.B. Smith and S. Chiranjeevi, J. Phys. Chem., 70, 3505 (1966).

7. W.-L. Li, Y.-P. Wang, Y.-B. Ji, Y.-Q. Fang and P. Zhao, China J. Chinese Mater. Med., 396 (2007).

8. S.W. Huang and W.-H. Wu, J. Chinese Med. Mater., 1352 (2006).

9. C.Q. Song, Z.-R. Zheng, D. Liu, Z.-B. Hu and W.Y. Sheng, Acta Botanica Sinica, 39, 1169 (1997).

10. O. Ghribi, D.A. DeWitt, M.S. Forbes, A. Arad, M.M. Herman and J. Savory, J. Aizheimers Dis., 3, 387 (2001).

11. Y.-S. Wang and Y. Wang, China J. Chinese Mater. Med., 961 (2006)

12. L. Ping, Tianjin Pharm., 9 (2002).

13. L. Bo-ting and L. Xiao-jin, Chin. Tradit. Herbal Drugs, 42 (1990).

14. Y.P. Su, Q.-H. Wang, Y.-B. Xun, Z.Z. Bai, G.Q. Zhao and J.J. Zuo, Heilongjiang Med. J., 850 (2012).

15. L. Tang, Y. Ma, C.-Q. Cai, J.-S. Li, Y. Liu, B. Wang and X. Yao, Acta Chinese Med. Pharmacol., 51 (2011).

16. P.-H. Xu, Y. Gao, X.-Q. Zhang, K. Xu and L. Zhang, Chinese Tradit. Patent Med., 390 (2009). 